Z Rheumatol 2020 · 79:609-610

https://doi.org/10.1007/s00393-020-00868-2

Angenommen: 11. August 2020

(c) Springer Medizin Verlag GmbH, ein Teil von Springer Nature 2020

Unter den Patienten mit wiederkehrenden Fieberschüben nichtinfektiöser oder maligner Ursache konnte lange Zeit nur denen mit einem Familiären Mittelmeerfieber (FMF) eine Diagnose zugeordnet werden und mit Colchicin auch eine Prävention der Krankheitsschübe und der AA(Amyloid-A-Protein)-Amyloidose angeboten werden. Man sprach hier von einem periodischen Syndrom, obwohl gerade das FMF keine echte Periodizität aufweist, sondern die Fieberschübe sich in unregelmäßigen Abständen wiederholen. Neben dem Fieber zeigen diese Patienten oft Befunde an inneren Organen wie Peritoneum und Pleura, der Haut und dem Bewegungsapparat, einen Beginn in der Kindheit und Familiarität. Damit ist das FMF das bekannteste Beispiel für sog. autoinflammatorische Erkrankungen, die in den letzten 20 Jahren durch klinische und molekulare Forschung definiert werden konnten. Im Gegensatz zu den Autoimmunerkrankungen wird bei Autoinflammation nicht das Selbst als Fremd erkannt, sondern der Fehler liegt in den die Entzündung vermittelnden Mechanismen selbst, sodass antigenunabhängig Fieber auftritt.

\section{》) Bei Autoinflammation tritt antigenunabhängig Fieber auf}

Diese Erkrankungen sind durch das regelmäßige oder unregelmäßige Auftreten von gleichartigen Schüben mit Fieber, Befunden an inneren Organen, muskuloskeletalen Symptomen und Hautausschlägen gekennzeichnet. Bei Patienten mit einer auffälligen Familienanamnese und einem Erkrankungsbeginn in der

\author{
N. Blank ${ }^{1} \cdot$ H.-I. Huppertz ${ }^{2}$ \\ ${ }^{1}$ Medizinische Klinik 5 und Amyloidosezentrum, Sektion Rheumatologie, Universitätsklinikum Heidelberg, \\ Heidelberg, Deutschland \\ ${ }^{2}$ Bremen, Deutschland
}

\title{
Autoinflammatorische Erkrankungen
}

frühen Kindheit finden sich häufiger als pathogen beschriebene Varianten in der molekulargenetischen Diagnostik, im Vergleich zu Patienten mit einer unauffälligen Familienanamnese und einem Symptombeginn im Erwachsenenalter. Es geben aber nicht alle Patienten mit nachgewiesenen pathogenen Varianten auch Fieberschübe an. Der Beitrag von Oskar Schnappauf stellt die aktuellen technischen Entwicklungen in der molekulargenetischen Diagnostik dar. Die Zahl der monogenetischen autoinflammatorischen Erkrankungen nimmt ebenso zu wie die Zahl neuer genetischer Varianten, wobei manchmal nicht sicher zwischen pathogen, fraglich pathogen oder polymorph unterschieden werden kann. Hier scheinen neben den klassischen dominant und rezessiv vererbten Erkrankungen auch atypische Verläufe, Mischformen, somatische Mosaike und De-novo-Mutationen eine Rolle zu spielen. Der aktuelle Kenntnisstand zur Diagnostik bei den monogenetischen Erkrankungen wird von Oskar Schnappauf dargestellt, und zudem unterbreitet er Vorschläge zur rationalen Nutzung der neuen Techniken.

In den vergangenen Jahren wurde untersucht, mit welchen Mechanismen die als pathogenen erkannten genetischen Varianten bei den monogenetischen Erkrankungen zur Auslösung der Entzündung führen. Der Beitrag von Tilmann Kallinich et al. beschreibt die krankheitsspezifischen Wege der Signaltransduktion bei verschiedenen autoinflammatorischen Erkrankungen. Bei einigen, wie z.B. dem Cryopyrin-assoziierten periodischen Syndrom (CAPS) führen die genetischen Varian- ten $\mathrm{zu}$ einer starken Überproduktion von Interleukin-1 $\beta$ (IL-1 $\beta$ ). Dementsprechend können diese Erkrankungen mit IL-1 $\beta$ blockierenden Medikamenten behandelt werden. Darüber hinaus beschreiben Tilmann Kallinich et al. auch Erkrankungen, die mit anderen Aktivierungsmechanismen (z. B. Interferone, Tumornekrosefaktor oder Transkriptionsfaktoren) assoziiert sind. Diese autoinflammatorischen Erkrankungen müssen durch die klinische Präsentation und die molekulargenetische Diagnostik von den IL-1 $\beta$-vermittelten Erkrankungen differenziert und mit anderen Therapieansätzen behandelt werden.

Neben den monogenetischen autoinflammatorischen Erkrankungen gibt es auch polygenetische Formen. Der Beitrag von Dirk Föll et al. stellt den Morbus Still als ein Beispiel einer als polygenetisch angesehenen autoinflammatorischen Erkrankung vor. Das klinische Spektrum reicht von der systemischen juvenilen idiopathischen Arthritis (sJIA, dem klassischen Still Syndrom) bis zum Still-Syndrom des Erwachsenen (AOSD). Hierbei scheinen die Mechanismen der Autoinflammation in der frühen Phase der Erkrankung und die Mechanismen der Autoimmunität mit Gelenkdestruktion eher in der späten Phase der Erkrankung zu dominieren. Möglicherweise kann durch effektive Behandlung der frühen Phase die Entwicklung zur Autoimmunität verhindert werden. Das Makrophagenaktivierungssyndrom (MAS) wird von Dirk Föll et al. als seltene, aber typische lebensbedrohliche Komplikation bei der sJIA und dem AOSD beschrieben. Ein MAS kann auch bei anderen entzündlichen Erkrankungen auftreten. Interessanterwei- 
se liegen der Gicht ähnliche Pathomechanismen mit Aktivierung des angeborenen Immunsystems wie dem Morbus Still zugrunde, bei der Gicht durch Harnsäurekristalle ausgelöst.

Eine weitere Komplikation einer anhaltenden und unregulierten Entzündungsreaktion ist die Amyloidose, die in den Beiträgen von Norbert Blank et al. und Kaya Veelken et al. beschrieben ist. Insbesondere bei hochentzündlichen Erkrankungen, häufigen Schüben und chronischen Erkrankungen muss an die Entwicklung einer Amyloidose gedacht werden. Bei den chronisch entzündlichen Erkrankungen müssen die AA-Amyloidose (Amyloidfibrillen aus Serum-Amyloid-A-Spaltprodukten) und die AL-Amyloidose (Amyloidfibrillen aus Immunglobulinleichtketten) zuverlässig differenziert werden. In dem Beitrag von Norbert Blank et al. wird der Zusammenhang zwischen AA-Amyloidose und Autoinflammation dargestellt. Das Therapieziel bei der AA-Amyloidose ist die Normalisierung der Entzündungsreaktion. Die wesentlich häufigere $\mathrm{AL}$ Amyloidose ist im Beitrag von Kaya Veelken et al. dargestellt. Sie spielt im Gegensatz zur AA-Amyloidose bei Autoinflammation keine wesentliche Rolle. Bei der AL-Amyloidose handelt es sich um eine monoklonale Erkrankung von B-Lymphozyten, die zu einer lokalen oder seltener einer systemischen ALAmyloidose führen kann. Risikofaktoren für die Entwicklung einer Amyloidose, Diagnostik und Therapiestrategien unterscheiden sich ganz wesentlich und werden in den beiden Beiträgen separat dargestellt.

In diesem Themenheft werden die aktuellen Erkenntnisse zur Genetik, Pathogenese, Diagnostik und Therapie von autoinflammatorischen Erkrankungen dargestellt. Eine Abgrenzung von den bekannteren systemischen Autoimmunerkrankungen ist notwendig, um eine gezielte Therapie durchführen zu können. Eine frühzeitige Diagnose erspart den oft kindlichen Patienten unnötige Leidenswege und bei Vorhandensein einer kausalen Therapie ist eine normale Entwicklung möglich. Das Erkennen von Komplikationen wie MAS und Amyloidose wird zusätzlich günstige Auswirkungen auf die Gesamtprognose dieser Erkrankungen haben.

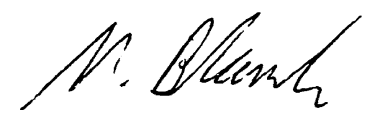

Prof. Dr. med. Norbert Blank

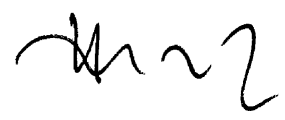

Prof. Dr. med. Hans-Iko Huppertz

\section{Korrespondenzadresse}

\section{Prof. Dr. N. Blank}

Medizinische Klinik 5 und Amyloidosezentrum, Sektion Rheumatologie, Universitätsklinikum Heidelberg

Im Neuenheimer Feld 410, 69120 Heidelberg,

Deutschland

norbert.blank@med.uni-heidelberg.de

\section{Prof. Dr. H.-I. Huppertz}

Schwachhauser Heerstr. 163a, 28211 Bremen, Deutschland

hihuppertz@hotmail.de

Interessenkonflikt. N. Blank gibt Advisory Boards und/oder Vorträge bei SOBI, Novartis, Roche, Chugai, MSD, Pfizer und UCB an. H.-I. Huppertz gibt keinen Interessenkonflikt an.
Seltene Infektionen der Lunge

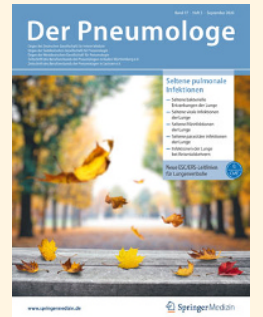

Im Jahr 2018 führten in Deutschland etwa siebenhunderttausend Fälle ambulant erworbener Pneumonie (CAP) zur stationären Aufnahme von fast

dreihunderttausend Patienten, von denen etwa $10-14 \%$ verstarben. Innerhalb der letzten Dekade stieg die Inzidenz der stationär behandelten CAP um achttausend Fälle pro Jahr - und dies noch vor dem sprunghaften Anstieg durch SARS-CoV-2. Für das Leitthema in Der Pneumologe 05/2020 haben erfahrene Autoren die relevantesten Fakten seltener Pneumonie-Erreger prägnant zusammengefasst und mit Fallvignetten angereichert. Damit erhalten Sie einen Überblick über ausgewählte seltene, aber wichtige Lungeninfektionen. Lassen Sie sich anregen zu detailliertem Nachlesen und nutzen Sie die Unterstützung für alltägliche differentialdiagnostische Überlegungen.

- Seltene bakterielle Erkrankungen

- Seltene virale Infektionen

- Seltene Pilzinfektionen

- Seltene parasitäre Infektionen

- Infektionen der Lunge bei Reiserückkehrern

Suchen Sie noch mehr zum Thema? Mit e.Med - den maßgeschneiderten Fortbildungsabos von Springer Medizin - haben Sie Zugriff auf alle Inhalte von SpringerMedizin.de. Sie können schnell und komfortabel in den für Sie relevanten Zeitschriften recherchieren und auf alle Inhalte im Volltext zugreifen.

Weitere Infos zu e.Med finden Sie auf springermedizin.de unter "Abos" 